\title{
Fetal methylmercury syndrome
}

INSERM

\section{Source}

INSERM. (1999). Orphanet: an online rare disease and orphan drug data base. Fetal methylmercury syndrome. ORPHA:1917

Foetal methylmercury syndrome is characterised by a group of symptoms that may be observed in a foetus or newborn when the mother was exposed during pregnancy to excessive amounts of methylmercury. 